\title{
Enfermedad de Behcet: reporte de caso y revisión de literatura
}

\section{Bechet's Disease: a case report and literature review}

Dra. Adriana Zúñiga Villegas. ${ }^{1}$ Dra. Marcela Kriebel Haehner ${ }^{2}$ Dr. Daniel Morún Calvo ${ }^{3}$ Dr. Luis Diego Monge Luna

1,2,3 y 4 Médico general. Trabajador independiente, San José Costa Rica.

Contacto: adrizuvi94@gmail.com

\section{Resumen}

La enfermedad de Behcet es una patología con múltiples afectaciones, siendo una de sus principales las úlceras orales y genitales. Su origen es desconocido, pero se cree que tiene una asociación genética. En el presente artículo, se reporta un caso clínico de una paciente femenina de 29 años de edad, diagnosticada en la adolescencia, con criterios mayores de diagnóstico como úlceras orales y genitales recurrentes, así como también criterios menores positivos. La paciente ha sido tratada con múltiples fármacos, tanto tópicos como sistémicos, para mejorar su calidad de vida. Aunque la enfermedad de Behcet es sumamente rara, es importante tener en cuenta el diagnóstico en pacientes con úlceras orales recurrentes. Sin el tratamiento adecuado, las manifestaciones clínicas de esta enfermedad pueden llegar a ser bastante severas y la calidad de vida de estos pacientes deteriorada.

\section{Palabras clave:}

Enfermedad de Behcet, úlceras orales, vasculitis sistémica

\section{Abstract}

Behcet's disease is a pathology with multiple affectations one of its main ones being oral and genital ulcers. Its origin is unknown but it is believed to have a genetic association. In this article we report a clinical case of a 29 -year-old female patient diagnosed in her teens with major diagnostic criteria such as recurrent oral and genital ulcers as well

Recibido: 21/oct/2020

Aceptado: $06 /$ nov/2020

Publicado: $15 /$ dic/2020 as minor positive criteria for Behcet's disease. The patient has been treated with multiple drugs both topical and systemic to improve her quality of life. Although Behcet's disease is extremely rare, it is important to keep this diagnosis in mind in patients with recurrent oral ulcers mainly to improve the quality of life of them, since without adequate treatment the clinical manifestations of this disease can become quite severe.

Keywords:

Behcet Disease, oral ulcers, systemic vasculitis 
Introducción

La enfermedad de Behcet es una patología multisistémica inflamatoria, crónica y recurrente. Es poco común y produce una inflamación a nivel de los vasos sanguíneos, por lo que algunos la denominan vasculitis sistémica. Su prevalencia se da en adultos jóvenes, generalmente durante la tercera y cuarta década de vida, aunque se ha descrito en la edad pediátrica. Es mayormente encontrada en países mediterráneos, del Medio Oriente y de Asia Oriental, por lo que se dice que su distribución geográfica sigue la Ruta de la Seda; sin embargo, se han reportado casos alrededor del mundo $(1,2,3)$.

Sus manifestaciones clínicas fueron descritas por primera vez alrededor del año 500 A.C por Hipócrates. No obstante, fue hasta 1937 que el dermatólogo turco Hulussi Behcet descubrió el síndrome con tres características principales: úlceras orales, úlceras genitales y afectación ocular. Se cree que esta enfermedad tiene predisposición genética, debido a que los pacientes suelen tener defectos inmunológicos y por lo general, presentan antecedentes familiares de enfermedades reumáticas $(2,3,4,5)$.

\section{Metodología}

Declaración ética: Este reporte fue aprobado por la paciente y no viola su privacidad. No fue necesario el consentimiento informado escrito, ya que se obtuvo consentimiento verbal. La búsqueda para la revisión de literatura se hizo en bases de datos como PubMed, UpToDate, DynaMed y Scielo. Los criterios de inclusión fueron publicaciones recientes, específicamente desde el año 2015 hasta la actualidad, con las palabras clave: "Enfemedad de Behcet", "úlceras orales" y "vasculitis sistémica", en el idioma español e inglés. Se revisaron 17 artículos que cumplen con los criterios esenciales sobre el tema.

\section{Reporte de Caso}

Paciente femenina de 29 años, costarricense, diagnosticada con enfermedad de Behcet hace 12 años en la ciudad de Nueva York, Estados Unidos. En edades tempranas, la paciente fue diagnosticada con Epilepsia Rolándica Benigna. Desde edades preescolares, comienza la sintomatología de la enfermedad de Behcet con úlceras orales y genitales, que fueron tratadas con antifúngicos sin respuesta a tratamiento. En ese entonces, se trataba con esteroides tópicos sin una respuesta adecuada. A los 10 años presenta empeoramiento de úlceras orales por lo que se inicia tratamiento con esteroides orales a altas dosis (con dosis de hasta $60 \mathrm{mg}$ diarios) sin mejoría clínica.

La paciente decide consultar un médico especialista en la Ciudad de Nueva York para una segunda opinión, en la cual se realiza el diagnóstico de Enfermedad de Behcet. Al momento, la paciente presentaba úlceras orales, eritema nodoso en miembros inferiores, prueba de patergia positiva y dolores articulares. Los resultados de laboratorios muestran antígeno B27 y B51 positivos. Desde el inicio de su diagnóstico, la paciente fue tratada con Azatioprina y Colchicina con buena respuesta al tratamiento ya que según evidencia científica era un adecuado tratamiento de primera línea (6).

Dos años después, la paciente fue declarada en remisión y su tratamiento suspendido. A los 21 años de edad, se reinició tratamiento con esteroides y Azatiopina por reaparición de úlceras orales y dolor articular en miembros inferiores. En 2012, la paciente presentó un episodio de lleitis Ulcerativa, para lo cual fue admitida en el hospital y se decidió iniciar tratamiento con Adalimumab y continuar con Azatiopina y deflazacort. Cursó con adecuada respuesta a tratamiento por tres años, hasta que en 2015 presentó fallo de respuesta a Adalimumab, se cambió a Certolizumab y no hubo mejoría de síntomas, por lo que se suspende en ese momento Certolizumab y se reinicia Adalimumab.

En enero del 2017, se decide cambiar el Adalimumab por Golimumab esperando una mejoría de síntomas, ya que la paciente continuaba con úlceras orales, artritis inflamatoria y fatiga crónica; sin embargo, se obtuvo un resultado adverso, con úlceras en faringe y esófago, las cuales se sobre infectaron y tuvieron que ser tratadas; por lo que nuevamente se decide regresar al tratamiento con Adalimumab, altas dosis de esteroides orales, Metrotexate y Azatriopina. Se continúa tratamiento de $25 \mathrm{mg}$ de metrotexate semanales, Adalimumab $40 \mathrm{mg}$ semanales, y $6 \mathrm{mg}$ de Deflazacort hasta febrero 2020, cuando la paciente consulta por segunda opinión en Minneapolis, Minnesota, ya que su sintomatología sigue siendo severa con artritis inflamatoria, úlceras orales, genitales y fatiga crónica. Se le cambia el tratamiento de Adalimumab por Apremilast, dado el éxito de los últimos estudios de este medicamento en pacientes con Behcet $(7,8)$.

Actualmente, la paciente se encuentra en tratamiento con Apremilast (tercer mes), y deflazacort. El cambio de Adalimumab a Apremilast le ha generado mejoría tanto en su fatiga crónica como en las úlceras orales; no obstante, persiste con úlceras genitales y artritis. La paciente refiere mejoría del $50 \%$ en su calidad de vida, siendo las úlceras genitales la mayor molestia en estos momentos.

El examen físico presenta cataratas bilaterales, soplo mitral sistólico grado II, dos úlceras genitales tipo fisuras de 
$1 \mathrm{~cm}$ cada una en vagina, dolorosas, pruriginosas, no sangran y sin descargas. No se ha evidenciado afectación renal ni neurológica. El plan a seguir es completar doce semanas de Apremilast para poder valorar respuesta a tratamiento de una manera adecuada.

Revisión de literatura

La enfermedad de Behcet, como se ha mencionado anteriormente, es una patología inflamatoria sistémica crónica, recurrente y remitente que es caracterizada por estomatitis aftosa recurrente, uveítis, úlceras genitales, lesiones cutáneas, afectación ocular, gastrointestinal, pulmonar y neurológica. Debido a que las manifestaciones clínicas son vasculares, se considera como una vasculitis. No obstante, las características histopatológicas que predominan en los tejidos inflamados son la infiltración de linfocitos y monocitos, en ciertas ocasiones leucocitos nucleares polimorfos, a través de pequeñas venas sin cambios microscópicos en las paredes de los vasos. También es común la trombofilia o tromboflebitis que afectan las venas pequeñas y grandes $(4,9,10,11)$.

Esta patología suele comenzar entre los 20-40 años, con una distribución homogénea entre hombres y mujeres. Sin embargo, el curso de la enfermedad es más grave en pacientes masculinos a edades más tempranas de inicio de síntomas, y un número mayor de órganos afectados al diagnóstico $(10,12,13)$.

La enfermedad tiene una mayor prevalencia en los países a lo largo de la antigua Ruta de la Seda desde Japón hasta la región mediterránea, con una mayor prevalencia en Turquía. Se ha visto una creciente población afectada en Europa y su aparición es principalmente esporádica; pero se ha visto agrupación de familias afectadas. Varios estudios han demostrado que el gen HLA-B51 está fuertemente asociado con el Bechet en diferentes grupos étnicos, encontrado en $60 \%$ de los casos (14). De todos modos, representa menos del $20 \%$ del riesgo genético, incluso en casos familiares (menos del $5 \%$ ), lo que indica que aún quedan por descubrir otros factores genéticos $(2,9,12,13$ 15,16).

\section{Tabla 1. Criterios Diagnósticos de la Enfermedad de Behcet}

\begin{tabular}{|l|l|}
\hline Criterios Mayores & Ulceración aftosa, $\geq$ tres episodios en 12 meses. \\
\hline Úlceras orales recurrentes & Úlceras aftosas \\
\hline Criterios Menores & Uveítis anterior o posterior / vasculitis retiniana \\
\hline Úlceras genitales recurrentes & Eritema nodoso, papulopustular, pseudofoliculitis \\
\hline Lesiones oculares & $\begin{array}{l}\text { Pequeña protuberancia roja o pústula, 24-48 horas después } \\
\text { de aplicar un pinchazo de aguja en el antebrazo. }\end{array}$ \\
\hline Lesiones cutáneas & Prueba de patergia positiva
\end{tabular}

FUENTE: Adaptado de la tabla 2 "Diagnostic criteria for Behçet's syndrome" tomado del artículo "Clinical manifestations and diagnosis of Behçet syndrome" Up to Date.

La patogénesis sigue siendo poco comprendida. Actualmente se piensa, como pasa en varios síndromes autoinmunes o autoinflamatorios, que ciertos factores infecciosos (virales o bacterianas, como Streptococcus sanguinis), inmunológicos y/o ambientales pueden desencadenar la sintomatología en individuos con variantes genéticas particulares, la reactividad cruzada con péptidos humanos y la activación del endotelio vascular. Además, es común la presencia de agentes gatillo, tanto físicos, ambientales o emocionales presentes al origen de la enfermedad o de una recurrencia. Contrario a otras enfermedades autoinmunes, el tabaquismo no se ha visto como un factor de riesgo en estos casos $(5,9)$.

El diagnóstico es comúnmente confirmado por descarte de otras enfermedades y se basa únicamente en síntomas clínicos. No existen pruebas de laboratorio que orienten específicamente a la patología. En sí, se realiza según los Criterios Internacionales de la Enfermedad de Behcet (ICBD), con una alta sensibilidad; listados en la Tabla 1. El paciente debe tener el criterio mayor, y al menos dos menores $(1,2,15)$.

Una prueba patognomónica es el fenómeno de patergia, una reacción de hipersensibilidad de los tejidos de la piel al trauma. La prueba positiva se observa cuando aparece de una pequeña protuberancia roja o pústula, 1 o 2 días después de un pinchazo de aguja en el antebrazo. Se ha visto que esta prueba tiene una alta sensibilidad y especificidad en pacientes turcos, algunos mediterráneos y del este medio $(5,12,15)$. 
El tratamiento es sintomático y su objetivo es reducir los síntomas, resolver la inflamación, limitar el daño tisular, reducir la recurrencia y prevenir las complicaciones asociadas; es decir, mejorar la calidad de vida del paciente. Este va a depender del sitio y gravedad de las lesiones, puede ser tanto tópico como sistémico. El tratamiento tópico se basa en aliviar molestias y lesiones ulcerativas dolorosas y para ello se utilizaron corticoides, antiinflamatorios no esteroideos o anestésico. En el caso de presentar alteraciones más graves se han empleado esteroides orales, agentes biológicos e inmunosupresores. Si existen complicaciones como una sobreinfección, se tratan acorde con la patología que se le diagnostique $(1,5,12)$.

\section{Discusión}

Hemos reportado un caso de una paciente con Enfermedad de Behcet, sus manifestaciones que incluyen aftas orales y genitales recurrentes, episodios severos de eritema nodoso, prueba de patergia positiva, un episodio de lleitis y HLA-B51 positivo. Ha sido tratada con altas dosis de esteroides, y con medicamentos biológicos como Adalimumab (medicamento que bloquea la interacción del factor de necrosis tumoral alfa con los receptores p55 y p75 en la superficie celular y causa cambios en los niveles de las moléculas de adhesión responsables de la migración leucocitaria), hasta los más nuevos como lo es el Apremilast (inhibidor de la fosfodiesterasa 4 que modula múltiples vías inflamatorias). Actualmente, la paciente reporta úlceras genitales recurrentes a pesar de tratamiento con Apremilast y Deflazacort. Sin embargo, dado el poco tiempo con el Apremilast, se debe esperar concluir 12 semanas de tratamiento. Nuestra paciente se encuentra con sintomatología leve, en comparación con otras etapas de su vida, no obstante, no se puede decir que el tratamiento es $100 \%$ exitoso ya que su enfermedad no se ha logrado controlar, lo que le genera deterioro en su calidad de vida. Si no se logra un tratamiento exitoso con Apremilast, se valorará un cambio de medicamento biológico nuevamente $(8,17)$.

En el 2018, la Liga Europea contra las Enfermedades Reumáticas (EULAR, por sus siglas en inglés) publicó una actualización de las recomendaciones que dieron en el 2008 para el manejo de la enfermedad de Behcet. En este, los especialistas describen 5 principios y 10 recomendaciones con base en resultados de diversas revisiones sistémicas y opiniones de expertos. Dentro de los principios, se postula que esta patología tiene un curso típico de recaídas y remisiones en donde el objetivo principal es suprimir lo más posible las exacerbaciones inflamatorias y por ende, los síntomas; característica que ha presentado nuestra paciente desde su diagnóstico. Además, hace mención a que el manejo debe de ser multidisciplinario e individualizado de conformidad con la edad, género, tipo y severidad del padecimiento. El compromiso ocular, vascular, neurológico y gastrointestinal puede ser asociado con mal pronóstico, por lo que la paciente presentada en este caso debe ser manejada muy de cerca para darle la mejor calidad de vida posible. Un dato favorable de esta enfermedad es que las manifestaciones clínicas mejoran con el tiempo en muchos pacientes (18).

Las recomendaciones del tratamiento presentadas en esta actualización van de la mano con los síntomas de los pacientes. En el caso de afectaciones mucocutáneas, se plantea el uso de medidas tópicas como esteroides cuando se presentan úlceras orales y genitales, y Colchicina como medida preventiva para la recurrencia de lesiones como eritema nodoso y úlceras genitales; se deben considerar fármacos como Azatioprina y Apremilast en casos seleccionados. En síntomas gastrointestinales, se recomienda una confirmación por estudios de imagen como endoscopía, para descartar úlceras, enfermedad inflamatoria intestinal e infecciones como tuberculosis. En el contexto de afectación gastrointestinal severa o refractaria, se deben considerar glucocorticoides cuando son exacerbaciones agudas con agentes que modifiquen la enfermedad como lo son la Azatioprina y el 5-ASA. Se debe considerar un manejo quirúrgico en caso de perforación, sangrado abundante o una obstrucción. Así mismo, cuando hay compromiso articular se debe iniciar Colchicina si es artritis aguda, glucocorticoides intraarticulares si es una enfermedad monoarticular y en casos crónicos o recurrentes tratar con Azatioprina, interferón-alfa o inhibidores del factor de necrosis tumoral alfa (18).

Se mencionan las recomendaciones publicadas relevantes con los síntomas que ha presentado nuestra paciente a lo largo del curso de su enfermedad. Los tratamientos que ha recibido han sido acorde con los aconsejados por los especialistas y siguen lo estipulado por esta guía. Lamentablemente, al ser una patología con exacerbaciones, no ha podido tener un período asintomático prolongado a pesar de recibir un buen manejo. No obstante, se espera que con el Apremilast se logren ver resultados favorables y así controlar su enfermedad de Behcet.

\section{Conclusiones}

La enfermedad de Behcet es una patología poco común y crónica que afecta diferentes órganos con un proceso inflamatorio. A pesar de que la edad más frecuente para el diagnóstico es entre la tercera y cuarta década de vida, se cuenta con un caso clínico que comenzó desde la edad pediátrica y se diagnosticó a los 17 años. La paciente ha presentado un curso largo con períodos recurrentes de úlceras orales, genitales y artritis principalmente. No obstante, ha presentado afectación 


\section{CIENCIA\&SALUD}

gastrointestinal a lo largo de su enfermedad. Se puede observar cómo la paciente cuenta con todas las características clínicas de la patología, incluyendo el gen HLA-B51; sin embargo, ha presentado dificultades con el tratamiento para conseguir una remisión total y mejorar su calidad de vida.

\section{Referencias bibliográficas}

1. Zeidan M, Saadoun D, Garrido M, Klatzmann D, Six A, Cacoub P. Behç et's disease physiopathology: a contemporary review [Internet]. Autoimmun Highlights (2016) 7:4. [Consultado el 14 de junio, 2020]. Disponible en: https://www.ncbi.nlm. nih.gov/pmc/articles/PMC4751097/

2. Smith EL, Yazici Y. Clinical manifestations and diagnosis of Behçet syndrome. Up to Date [Internet]. Actualizado el 14 de Febrero 2020, consultado el 12 Junio, 2020.

3. Tarquinio Marinho KC, Vieira Caputo B, Araujo Noro-Filho G, Magdalena Giovani E. Behçet's syndrome: Literature review and clinical case report [Internet]. rev esp cir oral maxilofac. 2016;38(1):105-110. [Consultado el 12 de junio, 2020]. Disponible en: $h t t p: / / s c i e l o . i s c i i i . e s / s c i e l o . p h p ? s c r i p t=s c i$ arttext\&pid=S1130-05582016000200009\&/ang=en

4. Solis Cartas U, Barbón Pérez OG, Lino Bascó E, Muñóz Balbín M, Molinero Rodríguez C, Solis Cartas E. Comportamiento clínico epidemiológico de la enfermedad de Behc, et [Internet]. rev colomb reumatol. 2016;23(2):79-84. [Consultado el 12 de junio, 2020]. Disponible en: http://www.scielo.org.co/pdf/rcre/v23n2/es_v23n2a03.pdf

5. Alfonso Valdés ME. ARTÍCULO DE REVISIÓN Síndrome de Behcet [Internet]. Revista Cubana de Hematología, Inmunol y Hemoter. 2016;32(3). [Consultado el 14 de junio, 2020]. Disponible en: http://www.revhematologia.sld.cu/index.php/hih/ article/view/425/246

6. Guedes-Barbosa, L. S. (2019). Oral and Genital Ulcers in Behçet's Disease. New England Journal of Medicine, 380(6). doi: 10.1056/nejmicm1802216

7. Hatemi, G., Mahr, A., Ishigatsubo, Y., Song, Y.-W., Takeno, M., Kim, D., ... Yazici, Y. (2019). Trial of Apremilast for Oral Ulcers in Behçet's Syndrome. New England Journal of Medicine, 381(20), 1918-1928. doi: 10.1056/nejmoa1816594

8. Hatemi, G., Melikoglu, M., Tunc, R., Korkmaz, C., Ozturk, B. T., Mat, C., ... Yazici, Y. (2015). Apremilast for Behçet's Syndrome - A Phase 2, Placebo-Controlled Study. New England Journal of Medicine, 372(16), 1510-1518. doi: 10.1056/ nejmoa1408684

9. Smith EL, Yazici Y. Pathogenesis of Behçet syndrome. Up to Date [Internet]. Actualizado el 10 de Diciembre 2019, consultado el 13 Junio, 2020.

10. Leccese P, Alpsoy E. Behçet's Disease: An Overview of Etiopathogenesis [Internet]. Front. Immunol., 10 May 2019. [Consultado el 14 de junio, 2020]. Disponible en: https://www.frontiersin.org/articles/10.3389/fimmu.2019.01067/full

11. Bittencourt Detanico A, Brandão ML, de Freitas Fernandes L, Ribeiro Camelo CP, Santana dos Santos JR. Aortic thrombosis in a patient with a late diagnosis of Behcet's disease [Internet]. J Vasc Bras. 2015 Apr.-June; 14(2):193-196. [Consultado el 14 de junio, 2020]. Disponible en: https://www.scielo.br/pdf/jvb/v14n2/1677-5449-jvb-14-02-00193.pdf

12. Rios Scherrer MA, Campos Garcia L, Barreto Rocha V. Behçet's disease: review with emphasis on dermatological aspects [Internet]. An. Bras. Dermatol. vol.92 no.4 Rio de Janeiro July/Aug. 2017. [Consultado el 14 de junio, 2020]. Disponible en: https://www.scielo.br/scielo.php?script=sci_arttext\&pid=S0365-05962017000400452\&lang=en

13. Esatoglu SN, Kutlubay Z, Ucar D, et al. Behçet's syndrome: providing integrated care [Internet]. J Multidiscip Healthc. 2017;10:309-319. [Consultado el 14 de junio, 2020]. Disponible en: https://www.dynamed.com/condition/behcet-syndrome\#GUID-5C112F5B-72FF-49AO-AF8F-AEE790CODAD3

14. DynaMed [Internet]. Ipswich (MA): EBSCO Information Services. 1995-.Record No. T114300, Behcet Syndrome; [actualizado 2019 Agosto 9]. [Consultado el 14 de junio, 2020]. Disponible en: https://www.dynamed.com/topics/dmp AN T114300.

15. Nair JR, Moot RJ. Behcet's disease [Internet]. Clinical Medicine 2017 Vol 17, No 1: 71-7. [Consultado el 14 de junio, 2020]. Disponible en: https://www.rcpjournals.org/content/clinmedicine/17/1/71

16. Jung Ryu H, Ryoung Seo M, Jin Choi H, Joo Baek H. Clinical phenotypes of Korean patients with Behcet disease according to gender, age at onset, and HLA-B51 [Internet]. Korean J Intern Med 2018;33:1025-1031. [Consultado el 14 de junio, 2020]. Disponible en: https://www.ncbi.n/m.nih.gov/pmc/articles/PMC6129630/pdf/kjim-2016-202.pdf

17. Adalimumab. (2018, Agosto 6). [Consultado el 14 de junio, 2020]. Disponible en: https://www.vademecum.es/principios-activos-adalimumab-104ab04

18. Hatemi G, Christensen R, Bang D, Bodaghi B, et al. 2018 update of the EULAR recommendations for the management of Behçet's syndrome [Internet]. Ann Rheum Dis 2018;77:808-818. [Consultado el 26 de octubre, 2020] Disponible en: https://ard.bmj.com/content/77/6/808?papetoc=

2020 Zúñiga Villegas et al. vol 4, número 6 Diciembre 2020 - Enero 2021 DOI: https://doi.org/10.34192/cienciaysalud.v4i6.227 